\title{
Exploring the scattering directionality and light interaction in nanoparticle dimers of different semiconductors
}

\author{
Ricardo Vergaz, ${ }^{*}$, Braulio García-Cámara ${ }^{1}$ J. Francisco Algorri, ${ }^{1}$ Alexander Cuadrado, ${ }^{2}$ and José M. Sánchez-Pena, ${ }^{1}$ \\ ${ }^{1}$ Group of Displays and Photonic Applications (GDAF-UC3M) Carlos III University of Madrid, 28911, Leganés, \\ Madrid, Spain, \\ ${ }^{2}$ Applied Optics Complutense Group. Department of Optics. University Complutense of Madrid. Faculty of Optics \\ and Optometry. Avda. Arcos de Jalón, 118. 28037 Madrid, Spain.
}

Received February 15, 2017; accepted May 22, 2017; published June 20,2017

\begin{abstract}
Assuming as a starting point our recent work on a dimer of silicon nanoparticles with light scattering directionality, we have explored the light interaction between the incoming and scattered electric fields in dimers made of other different semiconductors. The scattering directionality is achieved by accomplishing Kerker's conditions. By directing the scattered light towards the gap of the dimer, interferential effects can be used to achieve high or low light intensities as a basis of all-optical nanoswitches. A comparison between dimers of different materials is shown.
\end{abstract}

The emergence of Mie resonances, either electric or magnetic, in low-absorption CMOS-compatible dielectric nanoparticles has opened a new way in the research on light control at the nanoscale and its potential applications. [1-3]

In our recent work [4], we designed a dimer of silicon nanoparticles, accomplishing Kerker's conditions of light scattering directionality, in such a way that either a hotspot or a "cold spot" can be achieved in the gap between both nanoparticles. This is made by combining properly the satisfaction of the minimum forward scattering condition (MF) and the zero-backward scattering condition (ZB) to produce the convenient directional scattering outwards or inwards the gap. In the latter case, interferences appear, either constructive or destructive, in the gap region. The scheme in Figure 1 shows two possible arrangements that are called direct $/ 0^{\circ}$ configuration, producing a lack of light in the gap, and the inverse $/ 180^{\circ}$ configuration, which generates not only an enhanced concentration of light scattering in the gap, but also interferential effects.

There are several geometrical parameters to play with to optimize the contrast between the hot and cold states, such as the shape and size of the particles or the distance between them. Once they are optimized, the required effects are satisfied at a certain wavelength, in such a way that by shifting it, directional conditions are not satisfied anymore. Thus, we can change from a high to a null intensity of the electric field in the gap, or vice versa, producing an actual all-optical nanoswitch. If particles are

*E-mail: rvergaz@ing.uc3m.es not spherical ones, like nanodisks, this effect can be also achieved by varying the incident polarization [5].

In [4] a quantitative parameter was defined to evaluate the performance of the nanoswitch.

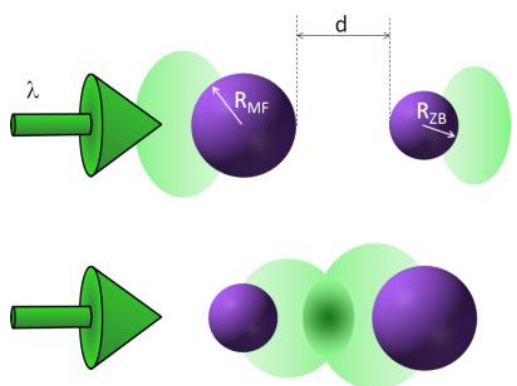

Fig. 1. Geometry of the dimer and the impinging light with the parameters used in this paper. Direct $/ 0^{\circ}$ (up) and Inverse $/ 180^{\circ}$ (down) configurations.

In this sense, a contrast parameter $\mathrm{CP}$ was defined as the ratio:

$$
C P=(\Delta E / \bar{E}) /(\Delta \lambda / \bar{\lambda}),
$$

where $\Delta \lambda=\lambda^{\prime}-\lambda_{0}$ and $\Delta E=E^{\prime}-E_{0}$. While $\lambda_{0}$ is the wavelength satisfying Kerker's conditions in the considered configuration, producing either a maximum or minimum intensity $\left(E_{0}\right)$ in the gap, $\lambda$ ' corresponds to such a state as Kerker's conditions are not satisfied and a contrary extreme of the intensity $\left(E^{\prime}\right)$ in the gap is found. Then, the contrast parameter $(C P)$ provides a quantitative measurement of the intensity contrast between the opposite states and their spectral closeness.

In this work, we have generalized our study searching the best contrast in dimers of nanoparticles for different semiconductor materials, using this metric. While silicon was explored in $[4,6]$, here we focus our attention on other interesting materials, like GaAs, Ge, $\mathrm{TiO}_{2}, \mathrm{AlAs}$, $\mathrm{AlSb}$ and GaP. Firstly, the sizes of nanoparticles of the different materials and the incident wavelength satisfying Kerker's conditions (the so-called target wavelength) were obtained through Mie theory as described in [4,7]. Then, the procedure is afterwards executed in two steps in 
this work. Once the components of the dimer are designed, numerical calculations employing Finite Element Method (COMSOLC) are carried out to determine the optimized gap distance, reinforcing the expected maximum /minimum of light in the gap through interferential effects in the inverse configuration. This first step determines the optimized geometry that provides an "ideal" maximum or minimum state. The second step, to find the opposite state of the switch, consists of a sweep in the wavelength around the target wavelength to find the optimum opposite behaviour in the gap region.

Figure 2 shows the dependence of the electric field intensity in the gap as a function of the gap distance $(d)$. Nanoparticles are made of different materials from one plot to another, and their sizes and wavelengths are tuned to accomplish Kerker's conditions for each case, corresponding to those appearing at Table 1, left columns. In every case, the total and scattered fields at the middle point of the gap are plotted as a function of the distance between nanoparticles, being $1 \mathrm{~V} / \mathrm{m}$ the incoming electric field intensity. Extending the range to long $d$ values, it can be seen clearly that when using the inverse $/ 180^{\circ}$ geometry a huge interferential effect appears. It is due to the interference between both scattered fields, which are directed towards the gap, and to the interference of the incoming field with those two scattered ones. The net effect of this interference is that the obtained maxima and minima are higher and deeper, respectively, than in the direct $/ 0^{\circ}$ geometry case. In every case, the first minimum at the total field (i.e., at shorter distances) is deeper than the rest and it will be selected for the next step. Although the interferential effect seems to increase for the next minimum, being deeper in the scattered field, the incoming field entering the gap reduces the net effect in the total electric field intensity. On the other hand, the first maximum is always the highest .

By comparing different materials and considering an inverse $/ 180^{\circ}$ configuration, $\mathrm{TiO}_{2}, \mathrm{AlAs}$ and $\mathrm{GaP}$ provide the best results. The highest maxima are obtained for these materials. In addition, they present deep minima in the scattered field, providing an almost totally destructive interference in the first two cases. In all the cases, the optimum gap distance to obtain the maximum field is in a range of 350-400nm, excepting $\mathrm{Ge}$ and $\mathrm{AlSb}$ that require larger distances.

Once the geometrical conditions have been fixed to work in the target wavelength obtaining a minimum in the gap, a sweep in the wavelength is performed around it to find the opposite behaviour. This is shown in Fig. 3 for some selected materials. The insets show the gap distance considered in any case, corresponding to the minimum electric field intensity in the gap for the inverse $/ 180^{\circ}$ geometry (minima at Fig. 2). Figure 3 shows three remarkable different results. GaAs nanoparticles (Fig. 3a) results in a bad situation, with no intense difference and a relatively large wavelength shift between the extreme positions. This situation is also observed considering Ge. A better result than the one for silicon [6] is obtained for $\mathrm{TiO}_{2}$ (Fig. 3b). In this case, the profile of the spectral dependence of light intensity in the gap region shows a remarkable valley, with a close maximum and minimum and with a high contrast. In fact, $C P$ is around 60 in this case, as can be seen in Table 1 . The best case is that of AlAs nanoparticles (Fig. 3c), which show a deep minimum (just a $15 \%$ of the incoming field) surrounded by two high maxima (1.7 times larger than the incoming field). $C P$ is over 100 in this case. In every case, a shift of the minimum is found when changing from a scattered field to a total field, due to the effect of the incoming field in the interference.

A summary of the optimum configurations of every considered material is included in Table 1 . In some cases, there is a slight shift between $\lambda_{0}$, and the value used in $\Delta \lambda$ due to the resolution of the simulation $(5 \mathrm{~nm})$ or a shift in $\lambda_{0}$, probably due to the interferential effects. The silicon dimer case [6] is shown in the first row for the sake of comparison. The CPs in GaAs and Ge materials, the latter one produced in the near infrared, are worse than the ones obtained for silicon. The reason is mainly the longer wavelength separation between maximum and minimum. On the other hand, a better result is achieved for $\mathrm{TiO}_{2}$, mainly due to the shorter wavelength range in which the effects can be found. AlAs is even better, as the combination of short wavelength separation and high contrast in electric field intensity makes it a very interesting candidate to be used in the proposed nanoswitch configuration. The results for $\mathrm{AlSb}$ and $\mathrm{GaP}$, although with apparent higher $C P$, show more peaks and noisy aspect, needing further study.

\begin{tabular}{|c|c|c|c|c|c|c|c|}
\hline Material & $\begin{array}{l}\mathbf{R}_{\mathrm{ZB}} \\
(\mathrm{nm})\end{array}$ & $\begin{array}{l}\mathbf{R}_{\mathrm{MF}} \\
(\mathrm{nm})\end{array}$ & $\begin{array}{c}\lambda 0 \\
(\mathrm{~nm})\end{array}$ & $\underset{(\mathrm{nm})}{\mathbf{d}}$ & $\Delta \mathrm{E}(\mathrm{V} / \mathrm{m})$ & $\Delta \lambda(\mathrm{nm})$ & CP \\
\hline $\mathrm{Si}$ & 82 & 96 & 700 & 120 & $\begin{array}{l}0.47-0.28 \\
0.28-1.75 \\
\end{array}$ & $\begin{array}{l}665-700 \\
700-770\end{array}$ & $\begin{array}{r}-12.65 \\
51.65 \\
\end{array}$ \\
\hline GaAs & 75 & 96 & 690 & 105 & $\begin{array}{l}0.87-0.32 \\
0.32-1.34\end{array}$ & $\begin{array}{l}605-685 \\
685-765\end{array}$ & $\begin{array}{l}-14.81 \\
27.43 \\
\end{array}$ \\
\hline Ge & 99 & 132 & 1079 & 160 & $\begin{array}{l}0.91-0.34 \\
0.34-1.48\end{array}$ & $\begin{array}{c}925-1075 \\
1075-1210\end{array}$ & $\begin{array}{r}-11.93 \\
26.74\end{array}$ \\
\hline $\mathrm{TiO}_{2}$ & 78 & 93 & 543 & 80 & $\begin{array}{l}1.01-0.24 \\
0.24-1.62\end{array}$ & $\begin{array}{l}510-545 \\
545-595\end{array}$ & $\begin{array}{l}-56.39 \\
62.53 \\
\end{array}$ \\
\hline AlAs & 82 & 98 & 600 & 95 & $\begin{array}{l}1.39-0.14 \\
0.15-1.68 \\
\end{array}$ & $\begin{array}{l}555-600 \\
600-660 \\
\end{array}$ & $\begin{array}{r}-104.55 \\
107.53 \\
\end{array}$ \\
\hline AISb & 96 & 117 & 800 & 130 & $\begin{array}{l}1.23-0.11 \\
0.11-1.81 \\
\end{array}$ & $\begin{array}{l}730-800 \\
800-870 \\
\end{array}$ & $\begin{array}{l}-114.1 \\
173.27 \\
\end{array}$ \\
\hline GaP & 93 & 111 & 692 & 105 & $\begin{array}{l}1.28-0.13 \\
0.13-1.78 \\
\end{array}$ & $\begin{array}{l}640-695 \\
695-760\end{array}$ & $\begin{array}{l}-110.9 \\
135.30 \\
\end{array}$ \\
\hline
\end{tabular}

Table 1. Summary of the main results of the analysed dimers in $180^{\circ}$ configuration. The radii of both particles, target wavelength, gap distance, electric field intensity in the two wavelengths stressed and the contrast parameter are shown for each material.

Summing up, we have demonstrated a procedure that wide opens the possibility of finding, theoretically, the right couple of nanoparticles sizes, and their relative distance, to build dimers of semiconductor particles that can act as all-optical nanoswitches. Both particles are made of the same material, but we have found the conditions for different usual semiconductor materials employed in the off-the-shelf electronics current market, revealing AlAs as the best candidate. 
This work has been supported by Ministerio de Economía y Competitividad of Spain (grants no. TEC2013-47342- C2-2- R and no.TEC2013-50138- EXP) and the R\&D Program SINFOTON S2013/MIT-2790 of the Comunidad de Madrid) and the funding from Agencia Estatal de Investigación (AEI) and Fondo Europeo de Desarrollo Regional (FEDER) for the Project TEC201677242- C3-1- R AEI/FEDER, UE.

\section{References}

[1] R. Gómez-Medina, B. García-Cámara, I. Súarez-Lacalle, F. González, F. Moreno, M. Nieto-Vesperias, J.J. Sáenz, J. Nanophoton. 5053512 (2011).

[2] B. Rolly, B. Stout, N. Bonod, Opt. Express 20, 20376 (2012).

[3] B. García-Cámara, R. Gómez-Medina, J.J. Sáenz, B. Sepúlveda Opt. Expr. 21, 23007 (2013).

[4] B. García-Cámara et al., J. Phys. Chem. C. 119, 19558 (2015).

[5] A.I. Barreda, H. Saleh, A. Litman, F. González, J.-M. Geffrin, F. Moreno, Nat. Commun. 8, 13910 (2017).

[6] R. Vergaz et al., IEE Phot. Jour., 8(3), 4501410 (2016).

[7] B. García-Cámara et al., IEEE Photon. Technol. Lett. 27(19), 2059 (2015).

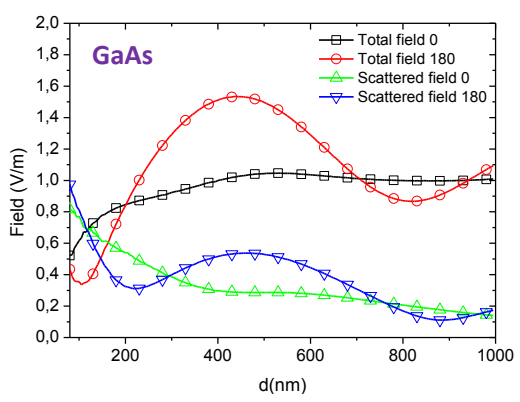

(a)

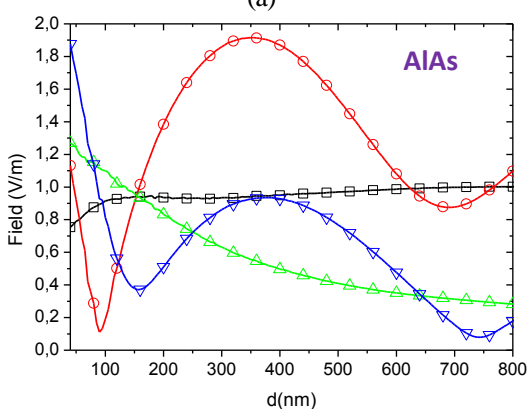

(d)

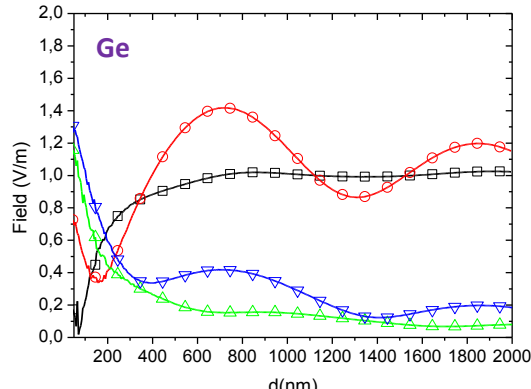

(b)

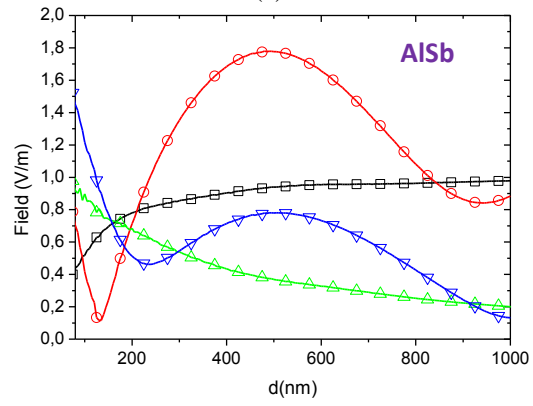

(e)

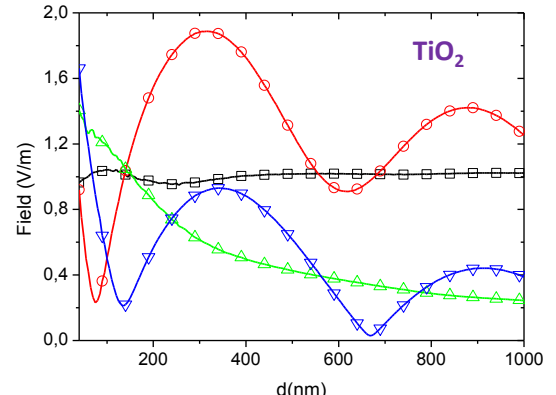

(c)

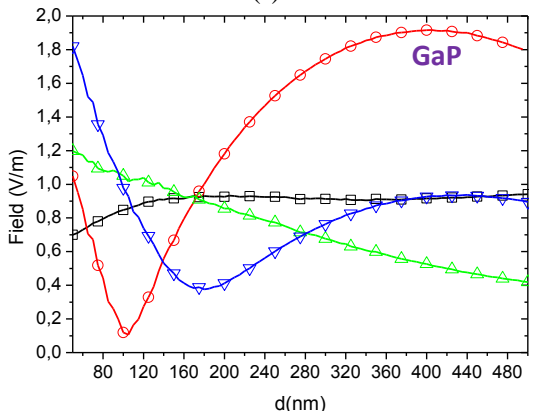

(f)

Fig. 2. Intensity of the electric field in the middle point of the gap for different geometries and materials, according to data of Table 1 . The total and scattered field (black squares and green triangles respectively) at direct $/ 0^{\circ}$ geometry and the total and scattered field (red circles and blue inverted triangles respectively) at inverse $/ 180^{\circ}$ geometry are plotted. Labels show the considered material.

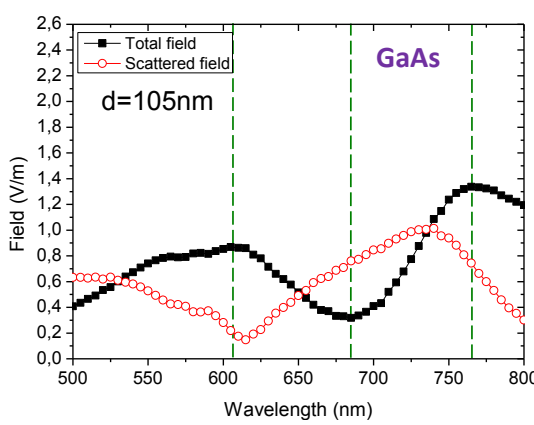

(a)

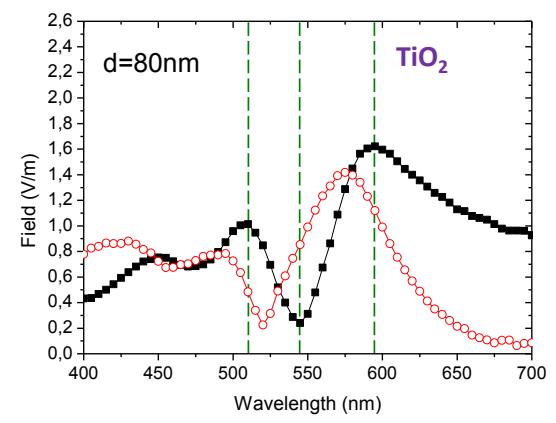

(b)

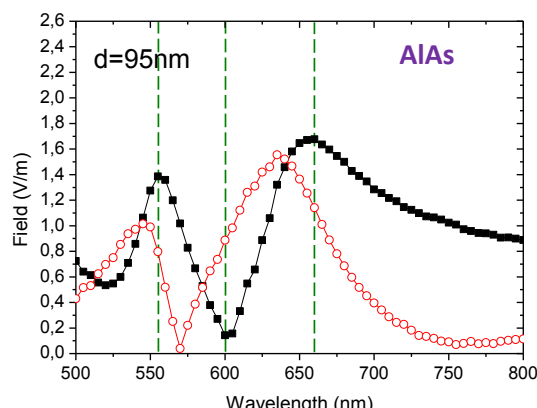

(c)

Fig. 3. Wavelength dependence of the intensity of the total (black squares) or the scattered (red hollow circles) electric field in the middle point of the gap for the inverse $/ 180^{\circ}$ geometry. The gap distance (left label) is the one producing the minimum of the total field for inverse $/ 180^{\circ}$ configurations in Fig. 2. Vertical lines correspond to the wavelengths of the local extremes used to compute CP of Table 1 , the one in the middle being the target wavelength position . Right labels show the considered material. 